\title{
Vértigo como presentación clínica de asa vascular de la arteria cerebelosa antero inferior
}

\section{Vertigo as a clinical presentation of a vascular loop of the anterior inferior cerebellar artery}

\author{
Yardany Rafael Méndez-Fandiño, Lina María López-SÁenz, \\ Carolina Moreno-Mancipe, Cristhian L. Ochoa Sanabria, \\ Diana K. Peñalosa-Villamizar, Angélica Johana Pérez-Hernández \\ - Tunja (Colombia)
}

\section{Resumen}

El vértigo es un frecuente motivo de consulta cuyo origen puede ser periférico o central; causas poco frecuentes de este último son las asas vasculares que afectan el VIII par craneal llevando a acúfenos, pérdida de la audición y mareo. Se presenta una mujer de 47 años de edad, quien consulta por un cuadro de mareo, náuseas, pérdida del tono postural, cefalea y parestesia facial, cuyo examen físico revela signos de vértigo periférico, iniciándose tratamiento con antieméticos, anticinetósicos y vasodilatadores que llevan a empeoramiento del cuadro, por lo que se sospecha de patología a nivel de sistema nervioso central (SNC) que ejerce compresión con la vasodilatación. Se realiza una resonancia magnética que muestra un asa vascular en la arteria cerebelosa inferior anterior (AICA) que ingresa al conducto auditivo interno (CAI) explicando la sintomatología y cuyo efecto compresivo de los nervios centrales genera un efecto paradójico con la toma de vasodilatadores.

(Acta Med Colomb 2018; 43: 226-229).

Palabras clave: vértigo, nervio vestibulococlear, vasodilatadores, compresión nerviosa, imagen por resonancia magnética.

\begin{abstract}
Vertigo is a frequent reason for consultation whose origin can be peripheral or central; infrequent causes of the latter are the vascular loops that affect the VIII cranial nerve leading to tinnitus, hearing loss and dizziness. The case of a 47-year-old woman who consulted for a picture of dizziness, nausea, loss of postural tone, headache and facial paraesthesia, and whose physical examination revealed signs of peripheral vertigo, is presented. Treatment was started with antiemetics, anticinetics and vasodilators that lead to worsening of the condition, for which pathology exerting compression due to vasodilation at the central nervous system (CNS) level, is suspected. An MRI is performed showing a vascular loop in the Anterior Lower Cerebellar Artery (AICA) that enters the Internal Auditory Canal (IAC) explaining the symptomatology and whose compressive effect of the central nerves generates a paradoxical effect with the taking of vasodilators. (Acta Med Colomb 2018; 43: 226-229).

Keywords: vertigo, vestibulocochlear nerve, vasodilators, nerve compression, magnetic resonance imaging.
\end{abstract}

\begin{abstract}
Dr. Yardany Rafael Méndez-Fandiño: Especialista en Medicina Interna y Epidemilogía Clínica. Magister en Investigación en Medicina Clínica. Director del Grupo de Investigación de Epidemiologia Clínica de Colombia (GRECO) y Grupo de Análisis de Resistencia Bacteriana de Boyacá (GRAB). Coordinador de Medicina Interna Universidad Pedagógica y Tecnológica de Colombia; Lina María López-Sáenz, Carolina Moreno-Mancipe, Cristhian L. Ochoa-Sanabria, Angélica Johana Pérez-Hernández: Estudiantes de Medicina, Universidad Pedagógica y Tecnológica de Colombia. Grupo de Investigación en Neurociencias - Universidad Pedagógica y Tecnológica de Colombia (GIN-UPTC; Diana K. Peñalosa-Villamizar: Estudiante de Medicina, Universidad Pedagógica y Tecnológica de Colombia. Tunja (Colombia).

Correspondencia: Diana Katherine PeñalosaVillamizar. Tunja (Colombia).

E-mail: dikas_28@hotmail.com

Recibido: 31/VIII/2017 Aceptado: 23/X/2018
\end{abstract}

\section{Introducción}

Dentro de los motivos de consulta más frecuentes, las alteraciones del equilibrio comprenden hasta $30 \%$ en la población general (1).El vértigo es una manifestación cortical producida por una alteración laberíntica o vestibular; el cual es definido como ilusión del movimiento del ambiente o del individuo, pudiendo originarse a nivel central o periférico
$(1,2)$. Kroenke et-al. encontraron que de los pacientes cuyo motivo de consulta fue vértigo, el $44 \%$ presentaban una vestíbulopatía de origen periférico, mientras $11 \%$ fue de origen central (3).

Se habla de vértigo periférico cuando está presente una lesión en el receptor o vías aferentes, las cuales incluyen las crestas ampollares o cualquier punto del laberinto pos- 
terior: utrículo, sáculo, ganglio de Scarpa del recorrido del nervio VIII. En este caso se suele presentar la triada clásica de hipoacusia perceptiva, acúfenos y vértigo; además de nistagmo y manifestaciones neurovegetativas (4). Cuando las estructuras afectadas en la etiología del vértigo implican el tallo cerebral, el cerebelo o las conexiones vestíbulocerebelosas, se habla de vértigo de origen central. Dentro de esta clasificación también se incluyen los cuadros clínicos que sean explicados por la afección de estructuras supratentoriales, como el tálamo o la corteza cerebral (5). Los tipos de lesiones que con mayor frecuencia causan vértigo central son los accidentes cerebrovasculares, malformaciones vasculares; tumores del ángulo pontocerebeloso, del tronco cerebral y del cerebelo; esclerosis múltiple, entre otros (2, 4). (Tabla 1) Por tanto, las malformaciones vasculares son las segundas en frecuencia como causa de vértigo central, de allí la importancia de su estudio.

Desde 1970 Janneta describió una entidad caracterizada por compresión de un nervio craneal por asas vasculares, denominándola compresión neurovascular; cuando ésta compromete el nervio vestibulococlear (VIII par craneal), es considerada una causa de acúfenos, perdida de la audición y vértigo (6), este último a veces incapacitante y causante de paroxismia vestibular, que se caracteriza por vértigo con o sin náuseas, acúfenos y pérdida de audición asociado a los cambios de posición (7).

\section{Caso clínico}

Mujer de 47 años de edad con antecedente de cáncer papilar de tiroides por el cual había sido sometida a cirugía de resección de la misma, y yodoterapia, que se encuentra en actual manejo con levotiroxina; quien ingresa al servicio de urgencias del Hospital Regional de Duitama (Colombia), por un cuadro clínico de 30 minutos de evolución, consistente en sensación de mareo, náuseas, con pérdida del tono postural acompañada de cefalea en hemicráneo

Tabla 1. Características vértigo central y periférico.

\begin{tabular}{|c|c|c|}
\hline Caracteristica & Central & Periférico \\
\hline Comienzo & Lento & Habitualmente brusco \\
\hline Morfología & Mareo mal definido & Vértigo \\
\hline Síntomas vegetativos & Escasos & Importantes \\
\hline Duración & Variable (días o años) & Episódico \\
\hline Evolución & Progresión frecuente & Raro más de una semana \\
\hline Hipoacusia & Excepcional & Habitual \\
\hline Oscilopsia* $^{*}$ & Importante & Escasa \\
\hline Síntomas asociados & Neurológicos deficitarios & Otológicos \\
\hline \multicolumn{3}{|c|}{$\begin{array}{l}\text { *Oscilopsia es la falsa percepción de que el mundo circundante se encuentra en mo- } \\
\text { vimiento. } \\
\text { Recuperado de: Rivera Rodríguez, T. Rodríguez Paradinas, M. Síndrome vertiginoso. } \\
\text { Medicine. 2007; 9(91): } 5857-5866\end{array}$} \\
\hline
\end{tabular}

derecho, cuyo inicio fue abrupto y de intensidad severa, $\sin$ vómito asociado. Al examen físico se encuentra álgica, con Romberg positivo y nistagmos horizontales. Se considera vértigo periférico o paroxístico benigno; pero se realiza tomografía axial computarizada (TAC) simple de cráneo, para descartar vértigo central por lesiones isquémicas o hemorrágicas supratentoriales. En el servicio de urgencias la paciente recibe tratamiento con betahistina, analgésicos y anticinetósicos, presentando una mejoría parcial.

Es evaluada por el servicio de otorrinolaringología considerando que se trata de vértigo con sensación de giro cefálico, asociado a síntomas vagales, náuseas y plenitud ótica izquierda, en presencia de tímpanos íntegros sin alteraciones en oído medio y orofaringe integra, con diadococinesis conservada, Romberg sin lateropulsión, Unterberguer con lateralización a la izquierda mayor a $45^{\circ}$, nistagmos espontáneos positivos con fase rápida a la derecha. Por lo anterior se sospecha vértigo periférico por hipovalencia vestibular izquierda, y se le solicita audiometría versal (logoaudiometría) y estudio vestibular con fotoelectronistragmografía, audiometría tonal e impedanciometría y le dan manejo ambulatorio con dimenhidrinato, metoclopramida, betahistamina.

Reingresa a los 18 días al servicio de urgencias por empeoramiento de los síntomas, con adormecimiento facial acompañado de malestar general, náuseas y síntomas disautonómicos. Al examen físico presenta lateralización de la marcha hacia la izquierda, aumento del polígono de sustentación, se le solicita (TAC) simple de cráneo, para observar compromiso cerebeloso, la cual no revela alteraciones. Se inicia manejo con vasodilatadores para mejorar los síntomas pero de forma paradójica éstos empeoran. Es llamativa la persistencia de síntomas paroxísticos, los cuales no se relacionan con movimientos anormales o con algún estímulo especifico, así que se considera que puede tratarse de un fenómeno de vasoespasmo, pero al suministrar vasodilatador empeora el cuadro.

Es revalorada por otorrinonaringología con los resultados de audiometría tonal, revelando normoacusia bilateral, timpanometría con curvas de tipo A normal bilaterales, al examen físico con otoscopia y rinoscopia normal, sin alteraciones otoneurológicas aparentes, diadococinesis conservada, sin evaluar Romberg y Unterberguer por limitación para la bipedestación de la paciente, nistagmos presentes multidireccionales, se palpan contracturas cervicales dolorosas al tacto y se sospecha más un cuadro por tensión muscular. Se realiza la resonancia magnética nuclear (RMN) cerebral contrastada, la cual revela un asa vascular en el ángulo pontocerebeloso izquierdo que ingresa al CAI en menos de 50\% de su longitud, entre el complejo del VIIVIII par craneal, sin angulación de éstos por asa vascular de tipo II, posiblemente de la AICA (Figura 1).

Actualmente la paciente refiere que a pesar de tratamiento sintomático, persiste el vértigo, y no hay claridad cómo manejar estos casos de vértigo. 

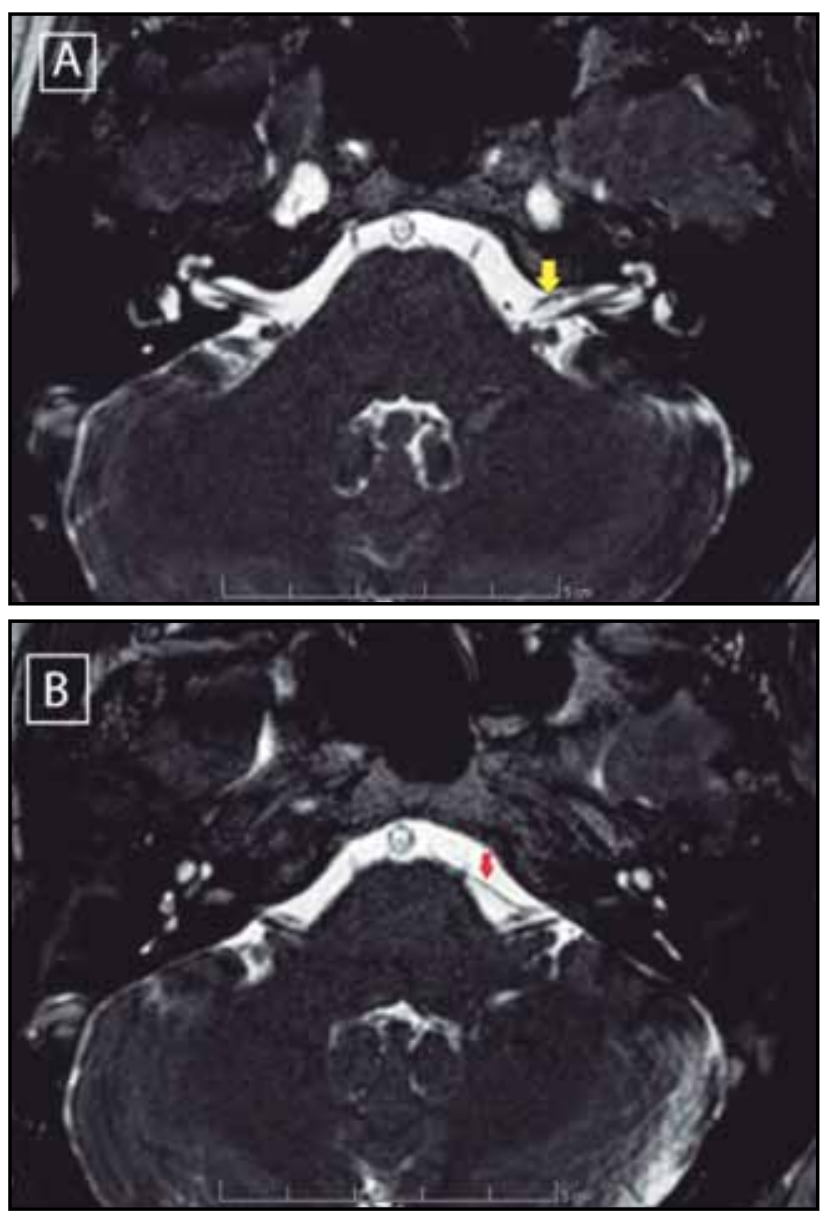

Figura 1.A: Imagen de resonancia magnética nuclear, en secuencia T2 CLEAR, en donde se aprecia el Asa Vascular (flecha amarilla) en el interior del conducto auditivo interno del lado izquierdo. B. Imagen de resonancia magnética nuclear, en secuencia T2 CLEAR, en la que se observa el recorrido del vaso sanguíneo (flecha roja) que da origen al asa vascular.

\section{Discusión}

El nervio facial y el vestíbulococlear emergen del ángulo pontocerebeloso que es un área rica en riego vascular y estructuras nerviosas; estos nervios durante su recorrido ingresan al CAI. Una de las estructuras vasculares que mantienen relación con estos es la AICA (6). En el CAI, hay un espacio mínimo para el nervio facial, vestíbulococlear y el bucle de la AICA, generando una susceptibilidad anatómica de compresión vascular, originando disfunción del nervio (8). Por ello, la compresión de nervios craneales por estructuras vasculares causa trastornos de los pares, de tal forma que si se comprime el nervio facial se presentará espasmo hemifacial; si se comprimen los troncos de los nervios auditivos y vestibulares podría originar pérdida de audición neurosensorial, acúfenos y vértigo $(8,9)$.

Las asas vasculares se presentan desde el nacimiento, y pueden ser asintomáticos o hacerse notorios clínicamente con el envejecimiento debido a la aterosclerosis, donde las paredes al ser más gruesas y rígidas pueden llegar a comprimir el nervio. De igual forma, los nervios craneales se estiran con el envejecimiento por disminución del lí-
Tabla 2. Clasificaciones de las asas vasculares.

\begin{tabular}{|l|l|}
\hline Clasificación anatómica (Chavda) & \multicolumn{1}{|c|}{ Clasificación según contacto } \\
\hline $\begin{array}{l}\text { TIPO I: } \\
\text { Situada sólo en el ángulo pontocerebe- } \\
\text { loso, pero no entra en el CAI }\end{array}$ & $\begin{array}{l}\text { Categoría A: } \\
\text { No hay contacto }\end{array}$ \\
\hline $\begin{array}{l}\text { Tipo II: } \\
\text { Entra, pero no se extiende a }>50 \% \text { de } \\
\text { la longitud de la CAI }\end{array}$ & $\begin{array}{l}\text { Categoría B: } \\
\text { Asa vascular que se extiende directamen- } \\
\text { te adyacente al nervio }\end{array}$ \\
\hline $\begin{array}{l}\text { TIPO III: } \\
\text { Se extiende }>50 \% \text { de la CAI }\end{array}$ & $\begin{array}{l}\text { Categoría C: } \\
\text { Bucle entre el VII y VIII nervio }\end{array}$ \\
\hline & $\begin{array}{l}\text { Categoría D: } \\
\text { Asa que desplaza el nervio dando lugar } \\
\text { a inclinación del nervio. }\end{array}$ \\
\hline $\begin{array}{l}\text { Recuperado de: Gorrie A., Warren III F.M., N. de la Garza A., Shelton C., Wiggins } \\
\text { III R. H. Is there a correlation between vascular loops in the cerebellopontine angle } \\
\text { and unexplained unilateral hearing loss?. Otology \& Neurology, Inc. 2009; 31:48-52. }\end{array}$ \\
\hline
\end{tabular}

quido cefalorraquídeo y por atrofia cerebral, llevando a un contacto anatómico entre una arteria previamente separada y el nervio (10).

Con la RMN cerebral es posible identificar detalles finos, tales como asas vasculares en el CAI $(10,11)$, de igual forma a partir de ella es posible visualizar estructuras vasculares y neuronales en el ángulo pontocerebeloso y determinar la relación entre el nervio vestíbulococlear y la vasculatura a su alrededor (8). En la Tabla 2 se observa la clasificación de las asas vasculares. Aproximadamente en $9 \%$ de los casos, las asas vasculares de la AICA se extienden más de 50\% de la longitud del CAI (10) y 12.3 $\%$ se producen dentro del CAI según estudios postmortem (11). Los principales síntomas ocasionados por una asa vascular de la AICA son acúfenos $(65 \%)$, con una incidencia de 7-12\% en la población general, y cuya forma de presentación en su mayoría es pulsátil (10).

Sirijci et al. analizaron 280 oídos de 140 personas con síntomas neurootológicos, de los cuales 20 (13.6\%) revelaron compresión vascular del VIII par por la AICA; los síntomas presentados fueron acúfenos 5 (25\%), vértigo $13(65 \%)$, pérdida de la audición $1(5 \%)$, pérdida de la audición y vértigo 1 (5\%) (6).

La cirugía de descompresión microvascular del VIII nervio en el ángulo pontocerebeloso, mediante aislamiento del nervio de la compresión por el vaso con fieltro de teflón o injertos de músculo, ha demostrado mejorar o eliminar los acúfenos, indicando que la sintomatología neurootológica se debe a un efecto directo de la compresión del nervio por las asas vasculares (12). A pesar de los hallazgos, aún es controvertido si las compresiones vasculares son la causa real de los síntomas neurootológicos; algunos artículos reportan que la demostración del contacto entre el asa vascular y el VIII nervio puede llegar a considerarse como un hallazgo anatómico normal y no ser el soporte diagnóstico del síndrome de compresión vascular. A pesar de esto, se ha sugerido una relación significativa entre el asa y el síndrome de compresión vascular $(6,13)$. 
A pesar de que las asas vasculares pueden causar compresión del VIII par craneal y las diferentes manifestaciones otológicas, aún hay controversia en cuanto a su fisiopatología y tratamiento de los síntomas neurootológicos. Sin embargo, en la actualidad la RMN ha sido una gran contribución para la investigación de la misma, ya que cuenta con múltiples ventajas al no ser invasiva y brindar visualización más detallada; aun así no se recomienda basar el diagnóstico únicamente en los hallazgos radiológicos, sino apoyarse también en los signos y síntomas descartando otras posibles causas. De igual forma, es importante tener en cuenta las asas vasculares como causa de vértigo al momento del tratamiento por el efecto negativo de los vasodilatadores en la mejoría clínica.

\section{Referencias}

1. Jalil J.M., Castro M.C., Pérez D.P., Sánchez D., Martínez D. Perfil clínico y poblacional de pacientes con vértigo. Clínica Occidente de Otorrinolaringologia, Medellín, Colombia. Acta otorrinolaringol. cir. cabeza cuello. 2014; 42(3): 152157.

2. Martínez-Vila E, Riverol Fernández $P$, Irimia Sieira P. Síndrome vestibular central. Rev Med Univ Navarra. 2003;47(4):51-9.

3. Kroenke K, Hoffman RM, Einstadter D. How common are various causes of dizziness?. A critical review. South Med J. 2000; 93: 160-7.

4. Rivera Rodríguez, T. Rodríguez Paradinas, M. Síndrome vertiginoso. Medicine. 2007; 9(91): 5857-5866. DOI:10.1016/S0211-3449(07)74747-1
5. Strupp M,Arbusow V.Acute vestibulopathy. Curr Opin Neurol. 2001; 14: 11-20.

6. Akif Sirikci,Yildirim Bayazit,Enver Ozer, Ayhan Ozkur, Ibrahim Adaletli, M. Ali Cüce, Metin Bayram. Magnetic resonance imaging based classification of anatomic relationship between the cochleovestibular nerve and anterior inferior cerebellar artery in patients with non-specific neuro-otologic symptoms. Surg Radiol Anat. 2005; 27: 531-535. DOI:10.1007/s00276-005-0015-6.

7. Wuertenberger CJ, Rosahl SK. Vertigo and Tinnitus Caused by Vascular Compression of the Vestibulocochlear Nerve, Not Intracanalicular Vestibular Schwannoma: Review and Case Presentation. Skull Base. 2009;19(6): 417-424. DOI: 10.1055/s-0029-1220209

8. Roldán-Fidalgo A., Rodríguez-Valiente A., Martín González F., Pinilla M., Trinidad A., Brea Álvarez B. Vascular loops and neuro-otologic symptoms: is there any correlation?. International Journal of Otolaryngology and Head \& Neck surgery. 2013; 2: 245-247. DOI:10.4236/ijohns.2013.26051

9. Gorrie A., Warren III F.M., N. de la Garza A., Shelton C., Wiggins III R. H Is there a correlation between vascular loops in the cerebellopontine angle and unexplained unilateral hearing loss?. Otology \& Neurology, Inc. 2009; 31: 48-52. DOI:10.1097/MAO.0b013e3181c0e63a

10.Gultekin S., Celik H., Akpek S., Oner Y., Gumus T., Tokgoz N. Vascular Loops at the Cerebellopontine Angle: Is there a Correlation with Tinnitus?. Am J Neuroradiol. 2008; 29: 1746-49. DOI:10.3174/ajnr.A1212

11. De Abreu Junior L, Kuniyoshi CH, Wolosker AB, et al. Vascular loops in the anterior inferior cerebellar artery, as identified by magnetic resonance imaging, and their relationship with otologic symptoms. Radiologia Brasileira. 2016; 49(5): 300-304. DOI: 10.1590/0100-3984.2015.0069

12. Ramly NA., Roslenda AR., Suraya A., Asma A. Vascular Loop in the cerebellopontine angle causing pulsatile tinnitus and headache: A case report. EXCLI Journal. 2014; 13:192-196

13. Borhei-Razavi H., Darvish O., Schick U. Disabling vertigo and tinnitus caused by intrameatal compression of the anterior inferior cerebellar artery on the vestibulocochlear nerve: a case report, surgical considerations and review of the literature. J Neurol Surg Rep. 2014; 75: e47-e51. DOI:10.1055/s-0033-1359299 